\title{
A REVIEW ON A COMPARATIVE STUDY ON EFFECTIVENESS AND SAFETY OF NOVEL ORAL ANTICOAGULANTS IN PATIENTS WITH ATRIAL FIBRILLATION
}

\section{Priyanka $\mathrm{P} \mathrm{K}^{1 *}$, Mathew George ${ }^{2}$, Lincy Joseph ${ }^{3}$}

${ }^{1}$ Department of Pharmacy Practice, Pushpagiri College of Pharmacy, Thiruvalla-689107, Kerala, India.

${ }^{2}$ Department of Pharmacology, Pushpagiri College of Pharmacy, Thiruvalla-689107, Kerala, India.

${ }^{3}$ Department of Pharmaceutical Chemistry, Pushpagiri College of Pharmacy, Thiruvalla-689107, Kerala, India.

\section{ABSTRACT}

Atrial fibrillation (AF) is characterized as an extremely rapid and disorganized atrial activation. These irregular heartbeats will cause blood to collect within the heart and potentially form a clot, which can travel to a person's brain and cause a stroke. AF increases stroke risk by 3 to 5 fold. Vitamin K antagonists (VKAs) are highly effective for the prevention of stroke, mainly of ischemic origin, in patients with AF. For this reason, VKAs are currently recommended in all AF patients at moderate to high risk for stroke or systemic embolism (SSE). VKAs have significant limitations, particularly their unpredictable anticoagulant response and numerous food and drug interactions, mandating regular laboratory monitoring. These limitations make treatment with VKAs problematic for many patients; as a result, only about half of all potentially eligible AF patients are treated with VKAs. Over the last several years, novel oral anticoagulant drugs (NOACs), including direct thrombin inhibitors (dabigatran) and factor Xa inhibitors (apixaban \& rivaroxaban), have been developed. New orally administered anticoagulant drugs have emerged as potential alternatives to VKAs for the prevention of ischaemic stroke or systemic embolism in patients with chronic atrial fibrillation. Novel oral anticoagulants (NOACs), due to their a lot of predictable therapeutic result and more favorable haemorrhagic risk profile, represent a particularly attractive therapeutic option in AF patients.

Keywords: Novel oral anticoagulants (NOACs), Vitamin K antagonist (VKAs), Atrial fibrillation, Apixaban, Dabigatran, Rivaroxaban.

\section{INTRODUCTION}

Atrial fibrillation is characterized as an extremely rapid and disorganized atrial activation. These irregular heartbeats can cause blood to collect within the heart and potentially form a clot, which may travel to a person's brain and cause a stroke. Atrial fibrillation increases stroke risk by 3-to 5fold. ${ }^{[1]}$ Strokes in AF are associated with greater mortality and morbidity, with more disability, longer hospital stays, and lowers rates of discharge to the patient's own home. Clearly, $\mathrm{AF}$ represents a major public health problem, with a significant impact on healthcare costs. ${ }^{[2]}$
Vitamin $\mathrm{K}$ antagonists (VKAs) are highly effective for the prevention of stroke, mainly of ischemic origin, in patients with AF. For this reason, VKAs are currently recommended in all AF patients at moderate to high risk for stroke or systemic embolism (SSE). VKAs have significant limitations, particularly their unpredictable anticoagulant response and numerous food and drug interactions, mandating regular laboratory monitoring. These limitations make treatment with VKAs problematic for many patients; as a result, only about half of all potentially eligible AF patients are treated with VKAs. 
Over the last several years, novel oral anticoagulant drugs (NOACs), including direct thrombin inhibitors (dabigatran) and factor $\mathrm{Xa}$ inhibitors (apixaban \& rivaroxaban), have been developed. These drugs have the potential to address some of the limitations of VKAs. These agents have fewer food and drug interactions and a lot of predictable anticoagulant effect, thus allowing fixed dosing without the need for laboratory monitoring. Furthermore, their shorter half-life may produce additional advantages, eg, if temporary interruption is required for a surgical procedure or in the case of a hemorrhagic complication.

NOACs have emerged as potential alternatives to VKAs for the prevention of ischaemic stroke or systemic embolism in patients with chronic atrial fibrillation, due to their more predictable therapeutic effect and a lot of favorable haemorrhagic risk profile, represent a particularly attractive therapeutic option in $\mathrm{AF}$ patients. ${ }^{[3]}$

Apixaban is a highly selective, orally bioavailable, and reversible direct inhibitor of free and clot-bound factor Xa. Factor Xa catalyzes the conversion of prothrombin to thrombin, the final enzyme in the coagulation cascade that is responsible for fibrin clot formation. ${ }^{[4]}$

Rivaroxaban competitively inhibits factor $\mathrm{Xa}$ and it does not require cofactors to exert its anticoagulant effect. It also inhibits both free and clot-bound factor $\mathrm{Xa}$ as well as prothrombinase activity, thereby prolonging clotting times. ${ }^{[5]}$

Dabigatran etexilate is a direct thromhin inhibitor that inhibits both free and fibrinbound thrombin. By inhibiting thrombin, it blocks the conversion of fibrinogen to fibrin and thus reduces clot formation. ${ }^{[6]}$

New oral anticoagulants reduced the risk for a composite end point of stroke and systemic embolism compared to VKAs. It was also found to be associated with a lower risk for key secondary efficacy outcomes, including ischemic and unidentified stroke, haemorrhagic stroke, all-cause mortality, and vascular mortality, compared to VKAs. We found no conclusive outcome with respect to major bleeding and gastrointestinal bleeding but found a substantial decrease in the risk for intracranial bleeding. Overall, results support the use of the new oral anticoagulants as alternatives to VKAs for long-term anticoagulation therapy in patients with AF. ${ }^{[7]}$

\section{REVIEW OF LITERATURES}

1. Alexandros Briasoulis et al ${ }^{[8]}$ (2018): conducted a study on "Safety and efficacy of novel oral anticoagulants versus warfarin in medical beneficiaries with atrial fibrillation (AF) and valvular heart disease (VHD)." Primary outcomes of all-cause mortality, ischemic strokes, major bleeding, and myocardial infarctions were compared across the 3 anticoagulants using 3-way propensitymatched samples. In this cohort of Medicare beneficiaries with VHD (excluding patients with prosthetic valves) and new-onset AF novel oral non-vitamin $K$ anticoagulants were safe and effective options for prevention of systemic thromboembolism.

2. Sebastian Schneeweiss et al ${ }^{[9]}$ (2012): conducted a study on "Comparative efficacy and safety of new oral anticoagulants in patients with atrial fibrillation." The study included 44535 patients enrolled in 3 trials of the efficacy of dabigatran, apixaban and rivaroxaban each compared with warfarin. We found no statistically vital effectualness variations among the three medications. Apixaban created considerably fewer major hemorrhages than dabigatran and rivaroxaban.

3. Amgad Mentias et al ${ }^{[1]}$ (2018): conducted a study on "Assessment of outcomes of treatment with oral anticoagulants in patients with atrial fibrillation and multiple chronic conditions." The study cohort included 21979 patients using dabigatran, 23177 using rivaroxaban and 101715 using warfarin In the propensity - matched cohorts, there were no differences in stroke rates between the 3 oral anticoagulant groups.

4. Simone Y Loo et al ${ }^{[10]}$ (2018): conducted a study on "Comparative effectiveness of novel oral anticoagulants (NOACS) in UK patients 
with nonvalvular atrial fibrillation (NVAF) and chronic kidney disease (CKD)." The study included 6818 adult patients newly treated with NOACs, matched 1:1 to new users of (Vitamin $k$ antagonist)VKAs on age, sex and high-dimensional propensity score. In the UK primary care, NOACs are overall effective and safe alternatives to VKAs, among patients with NVAF altogether, as well as in patients with NVAF and CKD.

5. Gianluigi Savarese et al [11] (2016): conducted a study on "Efficacy and safety of novel oral anticoagulants in patients with atrial fibrillation and heart failure". Four phase III clinical trials comparing NOACs to warfarin in patients with AF were enclosed. NOACs considerably reduced SSE, major bleeding, and intracranial hemorrhage in HF patients. No interactions in effectiveness and safety of NOACs were discovered between AF patients with and without HF.

6. Brandon K. Martinez et al [12] (2018): conducted a study on "Effectiveness and safety of apixaban, dabigatran, and rivaroxaban Versus warfarin in frail patients with nonvalvular atrial fibrillation". Users of apixaban, dabigatran, or rivaroxaban were individually 1:1 matched to warfarin users via propensity scores. Study found that rivaroxaban but not apixaban or dabigatran to be associated with reduced SSE versus warfarin in frail nonvalvular atrial fibrillation patients.

\section{CONCLUSION}

NOACs were comparable to VKAs in the prevention of ischemic stroke and systemic embolism, without being associated with an overall increased risk of serious adverse events. NOACs were found to have a lower risk of intracranial bleeding, and a higher risk of gastrointestinal bleeding. In patients with atrial fibrillation, apixaban was associated with lower risks of both stroke and major bleeding, dabigatran was associated with similar risk of stroke but lower risk of major hemorrhage, and rivaroxaban was related to similar risks of both stroke and major hemorrhage. We can conclude that NOAC are overall effective and safe alternatives to VKA for the prevention of ischemic stroke/SE in AF.

\section{REFERENCE}

1. Amgad Mentias, Ghanshyam Shantha, Pulkit Chadhury, Mary. S Vaughan Sarrazin ; Assessment of outcomes of treatment with oral anticoagulants in patients with atrial fibrillation and multiple chronic condition ; Journal of the American Medical Association Network Open ; Sep 2018; 1 (5) ; 1-16.

2. Gregory Y.H Lip, Can We Predict Stroke in Atrial Fibrillation? ; Clinical Cardiology; Jan 2012; 35 (S1); 21-27.

3. Francesco Dentali, Nicoletta Riva, Mark Crowther, Alexander G.G. Turpie, Gregory Y.H. Lip, Walter Ageno ; Efficacy and Safety of the Novel Oral Anticoagulants in Atrial Fibrillation ; Journal of the American Heart Association ; Nov 2012 ; 126 (20) ; 23812391.

4. Jimenez D1, Yusen RD, Ramacciotti E; Apixaban: an oral direct factor-Xa inhibitor; Advances in Therapy; Mar 2012; 29 (3); 187-201.

5. Meyer Michal Samama ; The mechanism of action of rivaroxaban - an oral, direct Factor Xa inhibitor - compared with other anticoagulants ; Thrombosis Research; June 2011 , 127 (6) ; 497-504.

6. Timothy Brighton; New oral anticoagulant drugs -mechanisms of action; Australian Prescriber; April 2010; 33; 38-41.

7. Shweta Bhatia, Supneet Sandhu and Dharinder Tayal; Safety and Efficacy of New Oral Anticoagulants in Patients with Atrial Fibrillation: Journal of Diabetic Complications \& Medicine, Nov 2015, 1(1), 1-8.

8. Alexandros Briasoulis, Chakradhari Inampudi , Emmanuel Akintoye, Paulino Alvarez, Sidakpal Panaic, Mary VaughanSarrazin ; Safety and efficacy of novel oral anticoagulants versus warfarin in medical beneficiaries with atrial fibrillation and valvular heart disease; Journal of the American Heart Association; Apr 2018; 7(8) ; 1-15. 
9. Sebastian Schneeweiss, Joshua J Gagne, Amanda R. Patrick, Niteesh K. Choudhry, Jerry Avorn ; Comparative efficacy and safety of new oral anticoagulants in patients with atrial fibrillation, circulation: cardiovascular Quality and Outcomes; July 2012 ; 5(4) ; 480-486.

10. Simone $Y$ Loo, Janie Coulombe, Sophie Dell Aniello, James M Brophy, Samy Suissa, Christel Renoux ; Comparative effectiveness of novel oral anticoagulants in UK patients with non valvular atrial fibrillation and chronic kidney disease ; British Medical Journal ; Jan 2018 ; 8(1) ; 1-9.

11. Gianluigi Savarese, Robert P. Giugliano, Giuseppe M.C. Rosano, John McMurray,
Giulia Magnani, Gerasimos Filippatos, Santo Dellegrottaglie, Lars $\mathrm{H}$. Lund, Bruno Trimarco, Pasquale Perrone-Filardi ; Efficacy and Safety of Novel Oral Anticoagulants in Patients With Atrial Fibrillation and Heart Failure; Journal of the American College of Cardiology: Heart Failure ; Nov 2016 ; 4(11) ; 870-880

12. Brandon K. Martinez, Nitesh A. Sood, Thomas J. Bunz, Craig I. Coleman ; Effectiveness and Safety of Apixaban, Dabigatran, and Rivaroxaban Versus Warfarin in Frail Patients With Nonvalvular Atrial Fibrillation; Journal of the American Heart Association; Apr 2018 ; 7(8) ; 1-10. 